\title{
ELLIPTIC SYSTEMS OF SINGULAR INTEGRAL OPERATORS. I. THE HALF-SPACE CASE( $\left.{ }^{1}\right)$
}

\author{
BY \\ ELIAHU SHAMIR
}

1. Introduction and statement of results. The problem studied in this paper is roughly the inversion of elliptic systems of singular integral equations in a halfspace of $R^{n}$. Ellipticity means that the system is invertible over the whole of $R^{n}$, in our case explicitly. The results with indication of proofs were announced in [14].

Let $(x, y)$ denote points in $R^{n}$ with $x \in R^{n-1}, y \in R . R_{+}^{n}\left[R_{-}^{n}\right]$ is the closed halfspace $y \geqq 0[y \leqq 0] . H^{s, p}$ denotes the space of distributions $u$ for which

$$
\|u\|_{s, p}=\left\|F^{-1}\left(1+|\xi|^{2}+\eta^{2}\right)^{s / 2} F u\right\|_{L^{p}}<\infty .
$$

Here $(F u)(\xi, \eta)=\int u(x, y) e^{i(x \cdot \xi+y \cdot \eta)} d x d y$ is the Fourier transform of $u(x, y)$ (also denoted by $\left.u^{\wedge}\right)$ and $(\xi, \eta)$ is dual to $(x, y)$. We assume throughout that $1<p<\infty$. The basic properties of $H^{s, p}$ are described in [1], [7]. Let $H_{-}^{s, p}$ denote the subspace of those $u \in H^{s, p}$ which are supported in $R_{-}^{n} \cdot H^{s, p}\left(R_{+}^{n}\right)$ is the quotient space $H^{s, p} / H_{-}^{s, p}$. (It is a space of distributions on $R_{+}^{n}$, the closed half-space.) $Y_{+}: H^{s, p} \rightarrow H^{s, p}\left(R_{+}^{n}\right)$ is the natural map onto the quotient (i.e., $Y_{+} u$ is the restriction of $u \in H^{s, p}$ to $R_{+}^{n}$ ), and $\left\|Y_{+} u\right\|_{s, p}$ is the quotient norm. $H_{+}^{s, p}, H^{s, p}\left(R_{-}^{n}\right)$ and $Y_{-}$are similarly defined. For $s=0$, we can identify $H_{ \pm}^{0, p}=L_{ \pm}^{p}$ with $L^{p}\left(R_{ \pm}^{n}\right)$, and $Y_{ \pm}$with multiplication by the characteristic function of $R_{ \pm}^{n}$. The definitions above extend to vector-valued functions componentwise.

Let $M(\xi, \eta)$ be a $N \times N$ matrix of functions, positively homogeneous of degree $0, C^{l+1}$ on the sphere $S^{n-1}=\left\{|\xi|^{2}+\eta^{2}=1\right\}$, where $l>n / 2$. The operator $M=F^{-1} M(\xi, \eta) F$ (whose symbol is $M(\xi, \eta)$ ) is bounded in $H^{s, p}$, invertible (elliptic) if $\operatorname{det}[M(\xi, \eta)] \neq 0$ on $S^{n-1}$. We consider the "mixed" operator $\tilde{M}$

$$
\tilde{\boldsymbol{M}}: H^{s, p} \rightarrow H^{s, p}\left(R_{-}^{n}\right) \times H^{s, p}\left(R_{+}^{n}\right), \quad \tilde{M} u=\left(Y_{-} u, Y_{+} M u\right) .
$$

To state the main results we introduce, for $0 \leqq \sigma<1$.

THE EIGenvalues CONDITION FOR $\sigma$. The eigenvalues $\lambda_{1}, \ldots, \lambda_{N}$ of the matrix $E=M^{-1}(0,1) M(0,-1)$ do not lie on the ray $(1 / 2 \pi) \operatorname{Arg} \lambda=1 / p+\sigma$.

Clearly this condition holds for all $0 \leqq \sigma<1$ except for at most $N$ exceptional values.

Presented to the Society, December 2, 1965 under the title Mixed estimates for systems of singular integral operators and applications to elliptic problems; received by the editors May 10, 1966.

(1) Partially supported by NSF grant GP-3940. 
TheOREM A. Let $s=k+\sigma, k$ integer, $0 \leqq \sigma<1$. There exist integers $k^{\prime \prime} \leqq k^{\prime}$ $\left(k^{\prime \prime}=k^{\prime}\right.$ in the scalar case) and a constant $C$ such that

$$
\|u\|_{s, p} \leqq C\left[\left\|Y_{-} u\right\|_{s, p}+\left\|Y_{+} M u\right\|_{s, p}\right], \text { for all } u \in H^{s, p}
$$

if and only if $k \geqq k^{\prime}$ and the eigenvalues condition for $\sigma$ is satisfied;

$$
\sum_{ \pm}\left\|V_{ \pm}\right\|_{-s, p^{\prime}} \leqq C\left\|V_{-}+M^{*} V_{+}\right\|_{-s, p^{\prime}}, \text { for all } V_{ \pm} \in H_{ \pm}^{-s, p^{\prime}}
$$

if and only if $k \leqq k^{\prime \prime}$ and the eigenvalues condition for $\sigma$ is satisfied. Here $M^{*}(\xi, \eta)$ is the conjugate transpose of $M(\xi, \eta)$ and $1 / p+1 / p^{\prime}=1$.

The estimate (1.2) means that $\tilde{M}$ is $1-1$ and has a closed range (i.e., left-invertible); the dual estimate (1.3) means that the same is true for $\tilde{M}^{*}$, the adjoint of $\tilde{M}$ acting between the adjoint spaces; hence $\tilde{M}$ itself is onto (i.e., right-invertible).

THEOREM $\mathrm{A}^{\prime}$. Let $\mathscr{N}^{s, p} \subset H^{s, p}$ denote the null space of $\tilde{M}, \mathscr{R}_{*}^{s, p} \subset H^{s, p}$ the range of $\tilde{M}^{*}$. Let

$$
J_{ \pm}^{l}=F^{-1}\left(\eta \pm i\left(|\xi|^{2}+1\right)^{1 / 2}\right)^{l} F
$$

Then for integral $l>0$

$$
\mathscr{N}^{s+l, p}=J_{-}^{-l} \mathscr{N}^{s, p} \cap J_{+}^{-l} \mathscr{N}^{s, p}, \quad \mathscr{R}_{*}^{s-l, p}=J_{+}^{l} \mathscr{R}_{*}^{s, p}+J_{-}^{l} \mathscr{R}_{*}^{s, p} .
$$

Similar formulas hold for $\mathscr{N}_{*}$ and $\mathscr{R}$.

COROLlARY A". Let $s=k+\sigma$. Then $\tilde{M}$ of (1.1) has a closed range (for any integer $k$ ) if and only if the eigenvalues condition for $\sigma$ is satisfied, which is the case for all $0 \leqq \sigma<1$ except for at most $N$ exceptional values.

In [11], [12] we have essentially obtained the above result for $n=1$ and general $M$ or $M=I$ (the identity matrix) and any $n \geqq 1$. In the later case, the exceptional values are $s=1 / p(\bmod 1)$. In fact, the present results show that $\tilde{M}$ for general $M$ behaves pretty much like $\tilde{\boldsymbol{I}}$.

The need to solve singular integral systems (with symbols homogeneous of degree 0 ) in half-spaces typically arise in connection with elliptic boundary value problems in domains $\Omega \subset R^{n+1}$ with discontinuities across $(n-1)$-dimensional manifolds of $\partial \Omega$ ("mixed" problems). The estimates of Theorem A yield, in a standard way, $L^{p}$ a priori estimates for mixed problems which imply that the associated operators are usually semi-Fredholm. Even $L^{2}$-estimates were obtained before only in special cases [8], [9], [10], and [18].

The estimates (1.2), (1.3) constitute the "potential theory" for elliptic systems of pseudo-differential operators [5] in subdomains $\Omega \subset R^{n}$. Again, standard arguments will show that these systems usually give rise to semi-Fredholm operators in $H^{s, p}$. The scalar $L^{2}$-case was recently discussed by Višik-Ėskin [19]; [20]. These questions and the applications to mixed elliptic problems will be discussed in part II of this paper. 
Let $M_{k}(\xi, \eta)=(\eta-i|\xi|)^{k} M(\xi, \eta)(\eta+i|\xi|)^{-k}$. Theorem $\mathrm{A}$ is derived from the following $L^{p}\left(R_{+}^{n}\right)$ result:

THEOREM B. There exists $k^{\prime \prime} \leqq k^{\prime}$ such that the operator

$$
Y_{+} u \rightarrow Y_{+} M_{k} Y_{+} u \text { in } L^{p}\left(R_{+}^{n}\right)
$$

is 1-1 and has a closed range iff $k \geqq k^{\prime}$ and the eigenvalues condition for $\sigma=0$ holds; it is onto iff $k \leqq k^{\prime \prime}$ and the eigenvalues condition for $\sigma=0$ holds.

THEOREM B'. The operator $Y_{+} u \rightarrow Y_{+} M Y_{+} u$ has a closed range in $L^{p}\left(R_{+}^{n}\right)$ iff the eigenvalues condition for $\sigma=0$ is satisfied.

The proof of Theorem B is given in $\$ \$ 2-5$. The argument becomes much simpler in the scalar case, $N=1$, or if $p=2$ (in particular if both $N=1$ and $p=2$, which is Višik-Ëskin case [19], [20]; they treat systems, $N>1$, only under a very restrictive and usually unverifiable condition on the matrix $M$ ). For $p=2, N \geqq 1$ we gave in [13] an immediate reduction to the 1-dimensional case, which in turn is treated in [11], [16]. Now for $N>1$ and any $p$ the crucial step is the following lemma, proved in $\$ 2$ :

LEMMA 1.1. Let $M_{\bar{\xi}}=F^{-1} M\left(\xi, \eta|\xi|^{-1}\right) F$. The $L^{p}$-estimate

$$
\left\|Y_{+} u\right\| \leqq C\left\|Y_{+} M Y_{+} u\right\|, \quad u \in L^{p}
$$

is equivalent to the family of estimates

$$
\left\|Y_{+} u\right\| \leqq C\left\|Y_{+} M_{\bar{\xi}} Y_{+} u\right\|, \quad u \in L^{p},
$$

for all $\xi$ satisfying $|\xi|=1$.

This is a "half-way" reduction to the 1-dimensional case. As usual with this type of problem, one tries to factor the symbol into a product of matrices holomorphic in $\operatorname{Im} \eta>0$ and $\operatorname{Im} \eta<0$. Here it suffices to factor (the one dimensional!) $M(\xi, \eta)$ for fixed $\xi$ and then substitute $\eta|\xi|^{-1}$ for $\eta$. We use results of GohbergKrein [2], [3] (described in \$3) which are applicable after the discontinuity of $M(\xi, \eta)$ at infinity is "filled in" ( $\$ 4$, with some details left to the appendix, $\S 7)$. Finally in $\S 5$ we get the a priori estimates for $Y_{+} M_{\bar{\xi}} Y_{+}$and prove Theorem B. The proof of Theorems $\mathrm{A}, \mathrm{A}^{\prime}, \mathrm{A}^{\prime \prime}$, and $\mathrm{B}^{\prime}$ are given in $\S 6$, which concludes with some additional observations on the operator $\tilde{M}$ is the spaces $H^{s, p}$ and other scales of spaces.

I am indebted to Professor Marvin Shinbrot for many stimulating conversations concerning this paper.

2. Multipliers; proof of Lemma 1.1. Let $m(\xi) \in L^{\infty}\left(R^{n}\right)$ and consider the operator $\mathbf{m} u=F^{-1} m(\cdot) F u, u \in C_{0}^{\infty}\left(R^{n}\right)$. Then $m(\xi)$ is an $L^{p}$-multiplier if $\mathbf{m}$ extends to a bounded operator in $L^{p}\left(R^{n}\right)$, the norm of which is denoted by $\|m\|_{M_{p}}$. 
Let $\alpha=\left(\alpha_{1}, \ldots, \alpha_{n}\right)$ be a multi-index. Let

$$
D^{\alpha} m=\frac{\partial^{|\alpha|} m}{\partial \xi_{1}^{\alpha} \cdots \partial \xi_{n^{n}}^{\alpha}}, \quad \xi^{\alpha}=\xi_{1}^{\alpha} \ldots \xi_{n^{n}}^{\alpha} ;|\alpha|=\sum \alpha_{j}
$$

unless otherwise stated, derivatives are taken in the distribution sense. $D^{\alpha}$ and $\alpha$ are called mixed if $\alpha_{j}=0$ or 1 . The following result is an improvement of MihlinHörmander multipliers theorem [4].

THEOREM 2.1 [15]. Let $l$ be an integer $>n / 2$ and

$$
\|m\|^{*}=\sum^{\prime} \operatorname{Sup}_{R_{i}>0} \int_{R_{i}<|x|<2 R_{i}}\left|\xi^{\alpha} D^{\alpha} m\right|^{2} \frac{d \xi}{R_{1} \cdots R_{n}}<\infty
$$

$\sum^{\prime}$ being taken over all mixed $\alpha,|\alpha| \leqq l$. Then $m$ is an $L^{p}$-multiplier for $1<p<\infty$. Moreover

$$
\|m\|_{M_{p}} \leqq C\|m\|^{*}, \quad C \text { depends only on } n \text { and } p \text {. }
$$

REMARK 2.2. Let $m_{\lambda}(\xi)=m\left(\lambda_{1} \xi_{1}, \ldots, \lambda_{n} \xi_{n}\right), \quad \lambda_{j}>0$. Then $\|m\|_{M_{p}}=\left\|m_{\lambda}\right\|_{M_{p}}$. (Actually this is true for any affine transformation in the $\xi$-space.) Also $\left\|m_{\lambda}\right\|^{*}=\|m\|^{*}$ and Theorem 2.1 is satisfactory in this respect. (This observation is actually used in deriving it from Hörmander's result, cf. [15].) Since

$$
\|m\|^{*} \leqq C \sum_{|\alpha| \leqq l}^{\prime} \operatorname{Sup}_{\xi \neq 0}\left|\xi^{\alpha} D^{\alpha} m\right|,
$$

the finiteness of the right-hand side is sufficient for $m$ to be an $L^{p}$-multiplier, and is more practical to check. If $m$ is homogeneous, it suffices to take Sup over $|\xi|=1$.

We switch back (and stay with) the notation of $\S 1$, i.e., $(x, y)$ and $(\xi, \eta)$ denote points in $R^{n}$ with $x$ and $\xi$ in $R^{n-1}, y$ and $\eta$ in $R$.

COROLlaRy 2.3. Let $q(\eta) \in C^{l}(R), l>n / 2$ and assume that $D^{k} q$ are bounded for $k \leqq l$ and

$$
\left(D^{k} q\right)(\eta)=O\left(|\eta|^{n-1-k-\delta}\right), \quad \delta>0 ; \quad|\eta| \rightarrow \infty .
$$

Then $q\left(\eta \cdot|\xi|^{-1}\right)$ is a multiplier for $1<p<\infty$.

(This follows easily from 2.2.)

A matrix of functions is a multiplier (i.e., gives rise to a bounded operator in $\left.L^{p} \times \cdots \times L^{p}\right)$ if and only if each entry is a multiplier. We consider now a positively homogeneous matrix: $M(\xi, \eta)$ which is invertible and in $C^{l+1}$ for $(\xi, \eta) \neq 0$, $l>n / 2$. Then $M$ and $M^{-1}$ are bounded in $L^{p} \times \cdots \times L^{p}$. The main part of the proof of Theorem $B$ consists in finding the conditions for the a priori estimate

$$
\left\|Y_{+} u\right\| \leqq C\left\|Y_{+} M Y_{+} u\right\|, \quad u \in L^{p},
$$

to hold. (\| $\|$ are $L^{p}$ norms.)

LEMMA 2.4. It is sufficient to obtain (2.3) for all $v$ which satisfy

$$
v^{\wedge}(\xi, \eta)=\beta(\xi) \wedge(\xi, \eta)
$$


where $u \in C_{0}^{\infty}$ and $\beta(\xi)$ is a smooth homogeneous function with spherical support (i.e., the support of $\beta$ on $|\xi|=1$ ) of diameter $\leqq 2 \delta$. ( $\delta>0$ is some preassigned number.)

Proof. We can find a $C^{\infty}$-partition of unity $1=\sum_{j=1}^{m} \beta_{f}(\xi)$ with $\beta_{j}(\xi)$ homogeneous functions with spherical support of diameter $\leqq 2 \delta$. For any $u \in C_{0}^{\infty}$

$$
Y_{+} u=\sum_{j=1}^{m} Y_{+} F_{x}^{-1} \beta_{j}(\xi) F_{x} u=\sum_{j=1}^{m} Y_{+} F^{-1} \beta_{j}(\xi) F u .
$$

Each $F^{-1} \beta_{j}(\xi) F u=u_{j}$ has the required form. Hence

$$
\left\|Y_{+} u\right\| \leqq \sum_{j}\left\|Y_{+} u_{j}\right\| \leqq \sum_{j} C\left\|Y_{+} M Y_{+} u_{j}\right\| \leqq C^{\prime}\left\|Y_{+} M Y_{+} u\right\| .
$$

The last inequality holds since the summation is finite and $\left(M Y_{+} u_{j}\right)^{\wedge}(\xi, \eta)$ $=\beta_{j}(\xi)\left(M Y_{+} u\right)^{\wedge}(\xi, \eta)$, each $\beta_{j}(\xi)$ being an $L^{p}$-multiplier. Thus (2.3) is established.

It is convenient now to introduce cylindrical coordinates $\left(|\xi|, \theta_{2}, \ldots, \theta_{n-1}, \eta\right)$ for points $P$ in $R^{n}$. They are related to the Cartesian coordinates $\left(\xi_{1}, \ldots, \xi_{n-1}, \eta\right)$ by $\xi_{j}=|\xi| \sin \theta_{j}, 2 \leqq j \leqq n-1$. We also denote by $S^{n-2}$ the unit sphere $|\xi|=1$ in $R^{n-1}$. Let $\xi \in S^{n-2}$ be fixed. The points

$$
\begin{aligned}
& P=(\xi, \eta)=\left(|\xi|, \theta_{2}, \ldots, \theta_{n-1}, \eta\right), \\
& \bar{P}=(\xi|\xi|, \eta)=\left(|\xi|, \bar{\theta}_{2}, \ldots, \bar{\theta}_{n-1}, \eta\right)
\end{aligned}
$$

are equidistant from the origin. Their angular separation is

$$
\left|\frac{P}{|P|}-\frac{\bar{P}}{|\bar{P}|}\right| \leqq \frac{|\xi|}{\left[\left(|\xi|^{2}+\eta^{2}\right)\right]^{1 / 2}} \cdot \sum_{j}\left|\theta_{j}-\bar{\theta}_{j}\right| \leqq \sum_{j}\left|\theta_{j}-\bar{\theta}_{j}\right|
$$

(clearly $\left.|\xi-\xi| \xi\right|^{-1}\left|\sim \sum\right| \theta_{j}-\tilde{\theta}_{j} \mid$ ). Let

$$
\Delta M=M(P)-M(\bar{P})=M(P /|P|)-M(\bar{P} /|\bar{P}|) .
$$

Since $M$ is in $C^{l}\left(S^{n-1}\right)\left(S^{n-1}\right.$ is the sphere $\left.|\xi|^{2}+\eta^{2}=1\right)$,

$$
\|\Delta M\|_{C^{l}\left(S^{n-1}\right)} \leqq C \sum_{j}\left|\theta_{j}-\tilde{\theta}_{j}\right| .
$$

Now we prove Lemma 1.1 (cf. §1), namely that (2.3) is equivalent to the family of $L^{p}$-estimates

$$
\left\|Y_{+} u\right\| \leqq C\left\|Y_{+} F^{-1} M(\xi|\xi|, \eta) F Y_{+} u\right\| .
$$

We notice first that, to establish (2.5) for a particular $\xi \in S^{n-2}$, it suffices to obtain it for all $v$ whose transform is of the form $v^{\wedge}(\eta, \xi)=\beta(\xi) u^{\wedge}(\eta, \xi)$, with $u \in C_{0}^{\infty}$ and for some $\delta$

$$
\beta(\xi)=\beta(\xi /|\xi|) \text { spherically supported in }|\xi /| \xi|-\xi|<\delta .
$$

Indeed, $M(\xi|\xi|, \eta)$ depends only on $|\xi|$ and $\eta$ and for such matrices the restricted estimates near one $\xi_{0}$ (say $\xi$ ) imply restricted estimates near any $\xi_{0} \in S^{n-2}$ (by 
obvious rotation argument), and by Lemma 2.4 this implies the unrestricted estimates (2.5). Invoking again Lemma 2.4 , this time for $M(P)=M(\xi, \eta)$, our lemma will be proved once we show that for a fixed $\xi$ and sufficiently small $\delta$, the restricted estimates for $M(P)$ and $M(\bar{P})$, with $\beta(\xi)$ restricted by $(2.6)$, are equivalent.

There is no loss of generality in taking $\xi=(1,0, \ldots, 0)$. We construct a family of homogeneous "cut-functions."

$$
\Gamma_{\delta}(\xi)=\Gamma\left(\xi_{1}, \xi_{2} / \delta, \ldots, \xi_{n-1} / \delta\right)
$$

where $\Gamma$ is a homogeneous $C^{\infty}$ function which is 1 for $|\xi-\xi|<\frac{1}{2}$, zero for $|\xi-\xi|<\frac{1}{2}$. The corresponding domains for $\Gamma_{\delta}$ shrink to $\xi$ as $\delta \rightarrow 0$ and for $\beta(\xi)$ restricted to the domain where $\Gamma_{\delta}=1$ we have $\Gamma_{\delta}(\xi) \beta(\xi)=\beta(\xi)$. Hence for any $M$ the restricted a priori estimate for $Y_{+} F^{-1} M F Y_{+}$is equivalent to the restricted one for

$$
Y_{+} F^{-1} \Gamma_{\delta}(\xi) M F Y_{+} .
$$

We need thus to show that for small $\delta$ these estimates for (2.7) with $M=M(P)$ and $M=M(\bar{P})$ are equivalent. This is clearly so if the difference $\Gamma_{\delta}(\xi)(M(P)$ $-M(\bar{P}))=\Gamma_{\delta}(\xi) \Delta M$ tends to 0 (as $\delta \rightarrow 0$ ) in the multipliers norm, which is the same for $\Gamma_{\delta}(\xi) \Delta M$ and $\Gamma(\xi) M\left(P_{\delta}\right)-M(\bar{P})$ where $P_{\delta}=\left(\xi_{1}, \delta \xi_{2}, \ldots, \delta \xi_{n-1}\right)$. Now $M\left(P_{\delta}\right)-M(\bar{P}) \rightarrow 0$ in $C^{l}$ and a fortiori in the multipliers norm, while $\Gamma(\xi)$ has a fixed norm. This concludes the proof.

3. Gohberg-Krein factorization. Let $\mathscr{A}=F L^{1}$, the ring of Fourier transforms of $L^{1}$-functions. The normed rings considered in this section are usually subrings of $\mathscr{A}$ with a bigger norm. A ring $R$ is called rationally dense if all the rational functions which vanish at $\infty$ and with poles off the real axis are in $R$, and are dense in $R$. A ring $R$ is decomposable if $R=R_{-} \oplus R_{+}$where $R_{+}\left[R_{-}\right]$is a closed subring consisting of all $f(\eta) \in R$ which admit holomorphic extension to $\operatorname{Im} \eta>0$ [Im $\eta<0] . \mathscr{A}$ itself is rationally dense and decomposable [2], [3], the projection $P_{+}$of $\mathscr{A}$ on $\mathscr{A}_{+}$is given by

$$
\left(P_{+} g\right)^{\wedge}(\lambda)=Y_{+}(\lambda) g^{\wedge}(\lambda) .
$$

To prove that a subring $R$ of $\mathscr{A}$ is decomposable, all we have to show is that $P_{+}$ is a bounded projection in $R$.

For $N \geqq 1, R_{N}$ is the ring of $N \times N$ matrices over $R$. Adjoining identity (the matrix $I$ ) we get the ring $I R_{N}=c I+R_{N}$. Let $O R_{N}$ be the group of invertible elements of $I R_{N}$. If $R \subset \mathscr{A}, O R_{N}$ consists of all matrices

$$
O R_{N}=\left\{c I+K(\eta) \mid \operatorname{det}\left[c I+K^{\wedge}(\lambda)\right] \neq 0\right\}, \quad-\infty \leqq \lambda \leqq \infty .
$$

(Notice that, taking $\lambda= \pm \infty$, (3.1) implies that $c \neq 0$. We shall usually take $c=1$.) Finally $c I+K(\eta) \in\left(O R_{N}\right)_{ \pm}$if the entries of $K$ are in $R_{ \pm}$and (3.1) holds. 
THEOREM 3.1 [2], [3]. Let $R$ be rationally dense and decomposable. Every matrix $M(\eta)=I+K(\eta) \in O R_{N}$ can be factored on the form

$$
M(\eta)=N_{-}^{-1}(\eta)((\eta-i) /(\eta+i))^{\kappa} N_{+}(\eta)
$$

where $N_{ \pm}(\eta)=I+K_{ \pm}(\eta)$ and $N_{ \pm}^{ \pm 1}(\eta) \in\left(O R_{N}\right)_{ \pm}$. The middle factor is a diagonal matrix

$$
\left(\frac{\eta-i}{\eta+i}\right)^{\kappa}=\operatorname{diag}\left[\left(\frac{\eta-i}{\eta+i}\right)^{\kappa_{1}}, \ldots,\left(\frac{\eta-i}{\eta+i}\right)^{\kappa_{N}}\right]
$$

with $\kappa_{1} \geqq \kappa_{2} \geqq \cdots \geqq \kappa_{N}$ (uniquely determined) integers called the partial indices of $M$. The sum $\kappa_{1}+\cdots+\kappa_{N}$ (the index of $M$ ) is stable (hence constant) under small perturbations of $M(\eta)$ (in the sup norm). It is given by

$$
\kappa_{1}+\cdots+\kappa_{N}=\int_{-\infty}^{\infty} d[\operatorname{Arg} \operatorname{det} M(\eta)]
$$

The partial sums $\kappa_{1}+\cdots+\kappa_{r}, r<N$, are upper-semicontinuous in $M(\eta)$, i.e., do not increase under small perturbations.

This result for such a wide class of rings was proved in [2], but for $M(\eta)$ defined on the unit circle $|\eta|=1$. However, the proof for the real line case is carried almost verbatim, in fact in this form it was first proved for $R=\mathscr{A}$ in [3]. The assertions about the sum and partial sums of the $\kappa_{j}$ are obtained by identifying

$$
\alpha=-\sum_{\kappa_{j}<0} \kappa_{j}, \quad \beta=-\sum_{\kappa_{j}>0} \kappa_{j}, \quad \alpha-\beta=-\sum \kappa_{j}
$$

respectively as the dimension of null space, codimension of the range and index of the Fredholm operator $Y_{+} F^{-1} M(\eta) F Y_{+}$in $L^{2}\left(R_{+}^{1}\right)$. The other partial sums are obtained by passing from $M(\eta)$ to $((\eta-i) /(\eta+i))^{k} M(\eta)$ for suitable $k$-a device we shall employ later (cf. Theorem B).

LEMMA 3.2. Let $R^{l} \subset \mathscr{A}$ consist of all $f(\eta)$ such that

$$
(\eta-i)^{k} D^{k} f(\eta) \in \mathscr{A} \quad 0 \leqq k \leqq l .
$$

Then $R^{l}$ is a decomposable, rationally dense normed ring with the norm

$$
\sum_{|k| \leqq l}\left\|(\eta-i)^{k} D^{k} f(\eta)\right\|_{A}=\sum_{|k| \leqq l} \int\left|\left(D_{\lambda}-i\right)^{k} \lambda^{k} f^{\wedge}(\lambda)\right| d \lambda .
$$

Proof. It is easily verified that $R$ is a normed ring, and the density proof is the same as for $\mathscr{A}$ in [3]. Now $P_{+}$is bounded in $R^{l}$ since

$$
\left(D_{\lambda}-i\right)^{k} \lambda^{k} Y_{+}(\lambda) f^{\wedge}(\lambda)=Y_{+}(\lambda)\left(D_{\lambda}-i\right)^{k} \lambda^{k} f^{\wedge}(\lambda)
$$

(in the distribution sense!), the $L^{1}$-norm of (3.8) is evidently $\leqq$ the $L^{1}$-norm of $\left(D_{\lambda}-i\right)^{k} \lambda^{k} f^{\wedge}(\lambda)$. 
4. Factorization of $M(\xi, \eta)$ for a fixed $\xi \neq 0$. The next goal is to factor $M(\xi, \eta)$ as a function of $\eta$ with respect to the ring $R^{l}$. However, in case $M(\xi, \infty) \neq M(\xi,-\infty)$ we have to fill in the jump at infinity. We start with some growth estimates for the derivatives of $M$.

LEMMA 4.1. Let $M(\xi, \eta) \in C^{l+1}\left(S^{n-1}\right)$ and let

$$
\lim _{\eta \rightarrow \pm \infty} M(\xi, \eta)=M(0, \pm 1)=E_{ \pm} .
$$

Then for $\xi \neq 0$

$$
D_{\eta}^{k}\left[M(\xi, \eta)-E_{ \pm}\right]=O\left(|\eta|^{-k-1}\right), \quad \eta \rightarrow \pm \infty ; 0 \leqq k \leqq l+1 .
$$

(This is simply $D_{\eta}^{k} M(\xi, \eta)$ for $k \geqq 1$.) The estimates are uniform for $|\xi|=1$.

Proof. Consider the case $\eta \rightarrow+\infty(\eta \rightarrow-\infty$ is similar $)$

$$
\begin{aligned}
M(\xi, \eta)-E_{ \pm} & =M\left(\xi \cdot \eta^{-1}, 1\right)-M(0,1) \\
& =\sum_{j} \frac{\partial M}{\partial \xi_{j}}(0,1) \frac{\xi_{j}}{\eta}+O\left(\eta^{-2}\right)=O\left(\eta^{-1}\right), \quad \eta \rightarrow \infty \\
D_{\eta}^{k} M(\xi, \eta) & =D_{\eta}^{k} M\left(\xi \cdot \eta^{-1}, 1\right)=O\left(|\eta|^{-k-1}\right), \quad \eta \rightarrow \infty
\end{aligned}
$$

If $M$ is invertible and $E_{+}=E_{-}$( $=I$ without loss of generality), it will be in $O R_{N}^{l}$ (see next lemma). Consider now the case $E_{+} \neq E_{-}$. To avoid technical complications now, assume in this section that

$$
E=E_{+}^{-1} E_{-} \text {is similar to } \operatorname{diag}\left[\lambda_{1}, \ldots, \lambda_{N}\right]
$$

(the general case is treated in the Appendix).

Without loss of generality we can take

$$
E_{+}=I, \quad E_{-}=E=\operatorname{diag}\left[\lambda_{1}, \ldots, \lambda_{N}\right] .
$$

All the $\lambda_{j}$ are nonzero. Define now $\zeta=\left(\zeta_{1}, \ldots, \zeta_{N}\right)$ by

$$
\zeta_{j}=\log \lambda_{j} / 2 \pi i=\sigma_{j}+i \tau_{j}, \quad-1+1 / p<\sigma_{j} \leqq 1 / p ; 1 \leqq j \leqq N
$$

such a choice of $\sigma_{j}$, all of them in an interval of length $<1$, is possible and determines $\zeta_{\text {j }}$ uniquely. Clearly

$$
\min \left(1-\sigma_{r}+\sigma_{j}\right)=\delta>0, \quad 1 \leqq j ; r \leqq N .
$$

In the sequel we consider fractional powers of $\eta \pm i$. In their definition we always use the principal branch of the $\operatorname{logarithm} \log z=\log |z|+i \operatorname{Arg} z,-\pi<\operatorname{Arg} z \leqq \pi$, which has a jump of $2 \pi$ across the negative real axis. With this in mind, and using scalar notation for diagonal matrices (cf. (3.3)) we set

$$
M_{*}(\xi, \eta)=(\eta-i)^{-\zeta} M(\xi, \eta)(\eta+i)^{\zeta} .
$$


Lemma 4.2. If $M \in C^{l+1}\left(S^{n-1}\right)$ is invertible, satisfies (4.4) and $\delta$ is given by (4.6) then

$$
D_{\eta}^{k}\left[M_{*}(\xi, \eta)-I\right]=O\left(|\eta|^{-k-\delta}\right), \quad \eta \rightarrow \pm \infty .
$$

This implies that $M_{*}(\xi, \eta) \in O R_{N}^{l}($ for $\xi \neq 0)$.

Proof. Write $M_{*}(\xi, \eta)$ as a sum of two terms

$$
\begin{array}{r}
M_{*}(\xi, \eta)=(\eta-i)^{-\zeta}\left[M(\xi, \eta)-E_{ \pm}\right](\eta+i)^{\zeta}+(\eta-i)^{-\zeta} E_{ \pm}(\eta+i)^{\zeta} \\
(+ \text { for } \eta>0, \quad-\text { for } \eta<0) .
\end{array}
$$

A typical entry of the first term is

$$
(\eta-i)^{-\zeta_{j}}\left[M-E_{ \pm}\right]_{g r}(\eta+i)^{\zeta_{r}}=O\left(|\eta|^{-1+\sigma_{r}-\sigma_{j}}\right)=O\left(|\eta|^{-\delta}\right)
$$

by (4.2), and this asymptotic relation is $(l+1)$-times differentiable (as long as (4.2) holds). The second term on (4.9) is diagonal, its $(j, j)$ entry is

$$
\begin{array}{ll}
(\eta-i)^{-\zeta_{j}} \cdot 1 \cdot(\eta+i)^{\zeta_{j}}=(\eta-i 0)^{-\zeta_{j}}(\eta+i 0)^{\zeta_{j}} \cdot 1 \cdot\left(1-i \eta^{-1}\right)^{-\zeta_{j}}\left(i+i \eta^{-1}\right)^{\zeta_{j}}, & \eta>0, \\
(\eta-i)^{-\zeta_{j}} \cdot \lambda_{j} \cdot(\eta+i)^{\zeta_{j}}=(\eta-i 0)^{-\zeta_{j}}(\eta+i 0)^{\zeta_{j}} \cdot \lambda_{j} \cdot\left(1-i \eta^{-1}\right)^{-\zeta_{j}}\left(i+i \eta^{-1}\right)^{\zeta_{j}}, & \eta<0 .
\end{array}
$$

In both cases, the first three terms on the right multiply up to 1 ; for $\eta<0$, this follows from the jump of the fractional powers across the negative real axis, and from (4.5). Expanding the last two factors in powers of $\eta^{-1}$ for $|\eta|>1$, we get

$$
(\eta-i)^{-\zeta} E_{ \pm}(\eta+i)^{\zeta}-I=\sum_{k=1}^{\infty} A_{k} \eta^{-k}, \quad|\eta|>1,
$$

which is obviously an infinitely differentiable asymptotic relation. Combining with (4.10), we get (4.8).

To prove $M_{*}(\zeta, \eta) \in O R_{N}^{l}$, it clearly suffices to show that for scalar functions $f(\eta) \in C^{l+1}$

$$
f^{(k)}(\eta)=O\left(|\eta|^{-k-\delta}\right), \quad k \leqq l+1 \text { implies }(\eta-i)^{k} f^{(k)}(\eta) \in \mathscr{A} ; \quad k \leqq l .
$$

If we denote $f_{k}=(\eta-i)^{k} f^{(k)}(\eta)$, then $f_{k}$ and $f_{k}^{\prime}$ are continuous, $f_{k}=O\left(|\eta|^{-\delta}\right)$, $f_{k}=O\left(|\eta|^{-1-\delta}\right),|\eta| \rightarrow \infty$. Hence $f_{k}^{\prime} \in L^{2}$. Passing to the transform, $\lambda f_{k}^{\wedge}(\lambda) \in L^{2}$ so that $f_{k}^{\wedge}(\lambda) \in L^{1}$ outside $(-\varepsilon, \varepsilon)$ for any $\varepsilon>0$. On the other hand $f_{k}^{\wedge}(\lambda)=O\left(\lambda^{\delta-1}\right)$ as $\lambda \rightarrow 0$ [17, Theorem 127] so this function is also in $L^{1}(-\varepsilon, \varepsilon)$. Thus $f_{k}$ itself is in $\mathscr{A}$ and the proof is concluded.

REMARK 4.3. The estimate (4.10) prefers some entries of $M_{*}-I$ over others. Indeed, since the second term in (4.9) is $I+O\left(|\eta|^{-1}\right)$, we have

$$
\left[M_{*}-I\right]_{j r}=O\left(|\eta|^{-1}\right)+O\left(|\eta|^{\sigma_{r}-\sigma_{j}-1}\right), \quad|\eta| \rightarrow \infty .
$$


Now let $B(\eta)$ be a bounded matrix function. Then

$$
\begin{aligned}
{\left[B\left(M_{*}-I\right)\right]_{q r} } & =\sum_{j} B_{q j}\left[M_{*}-I\right]_{j r}=\sum_{j} O\left(|\eta|^{\sigma_{r}-\sigma_{q}+\sigma_{q}-\sigma_{j}-1}\right)+O\left(|\eta|^{-1}\right) \\
& =O\left(|\eta|^{\sigma_{r}-\sigma_{q}-\delta}\right)+O\left(|\eta|^{-1}\right), \quad|\eta| \rightarrow \infty .
\end{aligned}
$$

Having proved that $M_{*} \in O R^{l}$, we can factor it by Theorem 3.1. For $M(\xi, \eta)$ itself we get:

THEOREM 4.4. Let $M(\xi, \eta) \in C^{l+1}\left(S^{n-1}\right)$ be homogeneous and invertible, and let it satisfy (4.4). For any fixed $\xi \neq 0$ we have (suppressing the dependence on $\xi$ )

$$
M(\xi, \eta)=Q^{-1}(\eta)(\eta-i)^{\xi}((\eta-i) /(\eta+i))^{x}(\eta+i)^{-\zeta} Q_{+}(\eta)
$$

where

$$
Q_{ \pm}(\eta)=(\eta \pm i)^{\zeta} N_{ \pm}(\eta)(\eta \pm i)^{-\zeta}
$$

$N_{ \pm}$and $\kappa$ are as in Theorem 3.1 (they arise from factoring $\left.M_{*}(\xi, \eta)\right) . Q_{+}(\eta)\left[Q_{-}(\eta)\right]$ and its inverse admit holomorphic extension to $\operatorname{Im} \eta>0[\operatorname{Im} \eta<0]$.

are bounded and

$$
Q_{ \pm}^{ \pm 1}(\eta) \in C^{l}(-\infty, \infty)
$$

$$
D^{r} Q_{ \pm}^{ \pm 1}(\eta)=O\left(|\eta|^{1-r-\delta}\right), \quad 1 \leqq r \leqq l .
$$

Moreover (reconsidering the dependence on $\xi$ ) all the $\kappa_{j}(\xi)$ are bounded on $|\xi|=1$.

COROLlary 4.5. There exist integers $k^{\prime}, k^{\prime \prime}$ such that for every $\xi \in S^{n-1}$ the partial indices $k+\kappa$, of

$$
M_{k}(\xi, \eta)=(\eta-i)^{k} M(\xi, \eta)(\eta+i)^{-k}
$$

are all $\geqq 0$ iff $k \geqq k^{\prime}$ and all $\leqq 0$ iff $k \leqq k^{\prime \prime}$.

Proof. The corollary follows from the last assertion of the theorem, which in turn follows from compactness and connectivity (or finiteness) of $S^{n-1}$, and the fact that $\kappa_{1}(\xi)\left[\kappa_{N}(\xi)\right]$ is upper- [lower-] semicontinuous (cf. Theorem 3.1, and note that $\kappa_{N}=\sum \kappa_{j}-\sum_{j=1}^{N-1} \kappa_{j}$, and that $\left.\kappa_{1} \geqq \kappa_{j} \geqq \kappa_{N}\right)$. Most other assertions of the theorem also follow from Theorem 3.1. To prove (4.14), notice that $(\eta-i)^{r} D^{r} N_{ \pm}^{ \pm 1}(\eta) \in \mathscr{A}$, hence $D^{r} N_{ \pm}^{ \pm 1}(\eta)=o\left(|\eta|^{-r}\right)$ as $|\eta| \rightarrow \infty$ and $r \leqq l . Q_{ \pm}$are given in (4.13) in terms of $N_{ \pm}$, and (4.14) follows easily (cf. the rightmost relation in (4.10)). It remains to show that $Q_{+}(\eta)$ is bounded (the argument for $Q_{-}, Q_{ \pm}^{-1}$ is the same). Now $\left[Q_{+}\right]_{r r}=\left[N_{+}\right]_{r r}$ and if $q \neq r$

$$
\left[Q_{+}\right]_{q r}=\left[N_{+}\right]_{q r} \cdot(\eta+i)^{\zeta_{q}-\zeta_{r}}=\left[K_{+}\right]_{q r}(\eta+i)^{\zeta_{q}-\zeta_{r}}
$$

where $N_{ \pm}=I+K_{ \pm}, K_{ \pm} \in R_{N}^{l}$.

The factorization (3.2) of $M_{*}=I+K$ easily yields

$$
K_{+}-K_{-}=\left[\left(\frac{\eta+i}{\eta-i}\right)^{x}-I\right] N_{-}+\left(\frac{\eta+i}{\eta-i}\right)^{x} N_{-} K
$$


since $N_{-}(\eta)$ is bounded, the first term on the right is $O\left(|\eta|^{-1}\right),|\eta| \rightarrow \infty$. Also $B(\eta)=((\eta+i) /(\eta-i))^{x} N_{-}(\eta)$ is bounded and $K=M_{*}-I$. Hence by (4.11)

$$
\left[K_{+}-K_{-}\right]_{q r}=O\left(|\eta|^{\sigma_{r}-\sigma_{q}-\delta}\right)+O\left(|\eta|^{-1}\right) .
$$

If $\operatorname{Re} \zeta_{q}=\sigma_{q} \leqq \sigma_{r}=\operatorname{Re} \zeta_{r},(4.16)$ is clearly bounded. If $\sigma_{q} \geqq \sigma_{r}$, (4.17) is at worst $O\left(|\eta|^{-\delta}\right)$. We get $\left[K_{+}\right]_{a r}$ from (4.17) by applying the projection $P_{+}$. It is known that if $-1 \leqq \gamma \leqq 0, \varepsilon>0$

$$
f \in C^{1} ; f(\eta)=O\left(|\eta|^{-\gamma}\right) \text { imply }\left(P_{+} f\right)(\eta)=O\left(|\eta|^{-\gamma+\varepsilon}\right) .
$$

We employ this for (4.17) with $\gamma=\max \left[-1, \sigma_{r}-\sigma_{q}-\delta\right]$. Using (4.16), we get

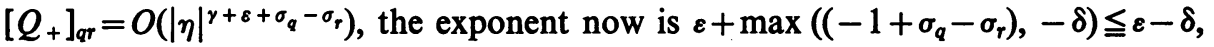
by (4.6). This is negative if $\varepsilon<\delta$. Thus $Q_{+}$is bounded.

COROLLARY 4.6. The matrices $Q_{ \pm}^{ \pm 1}\left(\eta|\xi|^{-1}\right)$ are $L^{p}$-multipliers in $R^{n}, 1<p<\infty$.

This follows from their boundedness, the estimates (4.14) and Corollary 2.3.

5. Proof of Theorem B. We pick now the line of argument from the end of $\$ 2$. It was shown there that the half-space a priori estimate for the symbol $M(\xi, \eta)$ is equivalent to the family of estimates for $M(\xi|\xi|, \eta)=M\left(\xi, \eta|\xi|^{-1}\right)$ with $|\xi|=1$. Substituting $\eta|\xi|^{-1}$ for $\eta$, Theorem 4.4 yields the factorization of $M\left(\xi, \eta|\xi|^{-1}\right)$ (again we suppress the dependence of $Q_{ \pm}$and on $\xi$ ).

$$
\begin{aligned}
M_{\bar{\xi}}(\xi, \eta) & =M\left(\xi, \eta|\xi|^{-1}\right) \\
& =Q_{-}^{-1}\left(\eta|\xi|^{-1}\right)(\eta-i|\xi|)^{\xi}\left(\frac{\eta-i|\xi|}{\eta+i|\xi|}\right)^{x}(\eta+i|\xi|)^{-\zeta} Q_{+}\left(\eta|\xi|^{-1}\right) .
\end{aligned}
$$

Consider now the operators

$$
M_{\bar{\xi}}=F^{-1} M_{\bar{\xi}}(\xi, \eta) F, \quad Q_{ \pm}=F^{-1} Q_{ \pm} F\left(Q_{ \pm}=Q_{ \pm}\left(\eta|\xi|^{-1}\right)\right),
$$

and

$$
D_{\kappa+\xi}=(\eta-i|\xi|)^{\xi}\left(\frac{\eta-i|\xi|}{\eta+i|\xi|}\right)^{\boldsymbol{x}}(\eta+i|\xi|)^{-\zeta}, \quad D_{x+\xi}=F^{-1} D_{\kappa+\xi} F .
$$

Let $u \in L^{p}$ be in the null space of $Y_{+} M_{\bar{\varepsilon}} Y_{+}$. Then $u_{+}=Y_{+} u$ is an $L^{p}$ function supported in $R_{+}^{n}$ and $F^{-1} Q_{-}^{-1} D_{x+\zeta} Q_{+} F u_{+}=u_{-}, u_{-}$supported in $R^{n}$, i.e., $\boldsymbol{D}_{\boldsymbol{x}+\zeta} \boldsymbol{Q}_{+} u_{+}=\boldsymbol{Q}_{-} \boldsymbol{u}_{-}$.

But $Q_{-}$is an $L^{p}$-multiplier holomorphic in $\operatorname{Im} \eta>0$. Hence (by Paley-Wiener) $\boldsymbol{Q}_{-} u_{-}=w_{-}, w_{-} \in L^{p}$. Similarly $\boldsymbol{Q}_{+} u_{+}=w_{+} \in L_{+}^{p}$. Thus $\boldsymbol{Q}_{+}$maps (boundedly) the null space of $Y_{+} M_{\xi} Y_{+}$into that of $Y_{+} D_{x+\xi} Y_{+}$. The process is clearly reversible $\left(Q_{+}^{-1}\right.$ maps back). We have proved the first half of

LEMma 5.1. $Q_{+}$sets an isomorphism between the null-spaces of $Y_{+} M_{\bar{\xi}} Y_{+}$and $Y_{+} D_{x+\xi} Y_{+}$. In particular both have the same dimension. $Q_{-}$sets an isomorphism between the ranges of these operators in $L^{p}\left(R_{+}^{n}\right)$. In particular the range of the first 
is closed if and only if the range of the second is closed, and both ranges have the same codimension.

To prove the isomorphism of the ranges, we observe that $Q$ - maps $L^{p}$ onto $L^{p}$ and $L^{p}$. onto $L^{p}$. Hence it induces an automorphism, still denoted by $Q_{-}$, of the quotient space $L^{p}\left(R_{+}^{n}\right)=L^{p} / L_{-}^{p}$ (also of $L_{+}^{p}$, which can be identified with $L^{p}\left(R_{+}^{n}\right)$; this is a special situation which is no more true for the general $H^{s, p}$ spaces considered in the next section). Let now $Y_{+} u \in L^{p}\left(R_{+}^{n}\right)$ be in the range of $Y_{+} M_{\xi} Y_{+}$. Then there is a $u_{+} \in L_{+}^{p}$ such that

$$
\begin{aligned}
\boldsymbol{Q}_{-}^{-1} \boldsymbol{D}_{\boldsymbol{x}+\zeta} \boldsymbol{Q}_{+} u_{+} & =\boldsymbol{v}\left(\bmod L_{-}^{p}\right), \\
\boldsymbol{D}_{\boldsymbol{x}+\zeta}\left(\boldsymbol{Q}_{+} u_{+}\right) & =\boldsymbol{Q}_{-} \boldsymbol{v}\left(\bmod L_{-}^{p}\right),
\end{aligned}
$$

since $Q$ - preserves mod $L_{-}^{p}$ (and is bounded, has bounded inverse in $L^{p}$ ). Now $Q_{+} u_{+} \in L_{+}^{p}$. So

$$
Y_{+} Q_{-} v=Q_{-} v\left(\bmod L_{-}^{p}\right)=Q_{-} Y_{+} v \quad\left(\bmod L_{-}^{p}\right)
$$

is in the range of $Y_{+} D_{x+\zeta} Y_{+}$. Clearly $Q_{-}^{-1}$ reverses the process and the lemma is proved.

The operator $Y_{+} D_{x+\xi} Y_{+}$is clearly the direct sum of the scalar operators $Y_{+} D_{x_{j}+\zeta_{j}} Y_{+}, 1 \leqq j \leqq N$. It will be shown in $\S 6$ that, if $\kappa_{j}$ is integer, $-1+1 / p$ $\leqq \operatorname{Re} \zeta_{j} \leqq 1 / p$,

$$
Y_{+} D_{x_{j}+\zeta_{j}} Y_{+} \text {has a closed range iff } \operatorname{Re} \zeta_{f} \neq 1 / p \quad(\bmod 1)
$$

and if (5.4) is satisfied, then

$$
Y_{+} D_{\kappa_{j}+\zeta_{j}} Y_{+} \text {is 1-1 iff } \kappa_{j}^{\prime} \geqq 0 \text {, onto iff } \kappa_{j} \leqq 0 .
$$

Clearly, these conditions should hold for each $j$ in order that $Y_{+} D_{x+\xi} Y_{+}$should have closed range, be 1-1 or onto, respectively.

Proof of Theorem B ( $(1) . M_{k}\left(\xi, \eta|\xi|^{-1}\right)$ has a factorization like (5.1) with partial indices $k+\kappa_{j}$ instead of $\kappa_{j}\left(Q_{ \pm}\right.$and $\zeta$ are the same). So by Lemma 5.1, (5.4) and (5.5), the conditions $k+\kappa_{j} \geqq 0$ and $\operatorname{Re} \zeta_{j} \neq 1 / p, 1 \leqq j \leqq N$, are necessary and sufficient for the estimate

$$
\left\|Y_{+} u\right\| \leqq C\left\|Y_{+} F^{-1} M_{k}\left(\xi, \eta|\xi|^{-1}\right) F Y_{+} u\right\|, \quad u \in L^{p}\left(R^{n}\right),
$$

which amounts to the operator in question being 1-1 and of closed range. By (4.5), $k+\kappa_{j} \geqq 0$ for every $|\xi|=1$ if and only if $k \geqq k^{\prime}$. Thus by (1.1), $k \geqq k^{\prime}$ and $\operatorname{Re} \zeta_{j} \neq 1 / p$, $1 \leqq j \leqq N$, are the necessary and sufficient conditions for

$$
\left\|Y_{+} u\right\| \leqq C\left\|Y_{+} F^{-1} M_{k}(\xi, \eta) F Y_{+} u\right\|, \quad u \in L^{p} .
$$

Notice that $\operatorname{Re} \zeta_{j} \neq 1 / p$ iff $\operatorname{Arg} \lambda_{j} \neq 2 \pi / p$ (cf. (4.1), (4.3), and (4.5)), i.e., iff the eigenvalues condition for $\sigma=0$ is satisfied. This proves one half of Theorem $B$. The other 
half (about $M_{k}$ being onto) is easily obtained by taking adjoints. If the matrix $M^{*}$ is the conjugate-transposed of $M$, then the adjoint of $Y_{+} M_{k} Y_{+}$in $L^{p}\left(R_{+}^{n}\right)$ is $Y_{+}\left(M^{*}\right)_{-k} Y_{+}$in $L^{p^{\prime}}\left(R_{+}^{n}\right)$, with $1 / p+1 / p^{\prime}=1$. The question is, then, when is the last operator 1-1 and of closed range. Now the factorization of $M_{k}(\xi, \eta)$ induces a similar factorization of $M_{-k}^{*}(\xi, \eta)$ with partial indices $-\kappa_{j}-k$ and fractional exponents $-\zeta_{j}$

$$
-1+1 / p^{\prime} \leqq \operatorname{Re}\left(-\zeta_{j}\right)<1 / p^{\prime}
$$

Again, $-k-\kappa_{j} \geqq 0$ (for all $|\xi|=1$ ) iff $k \leqq k^{n}$ for suitable $k^{\prime \prime}$. The condition for closed range is by (5.4) that $-\operatorname{Re} \zeta_{j} \neq 1 / p^{\prime}(\bmod 1)$ or $\operatorname{Re} \zeta_{j} \neq 1 / p$ as before. This concludes the proof of Theorem $B$.

REMARK 5.2. Notice that the same argument proves the second half of (5.5) from the first half.

6. The operators $\tilde{M}, \tilde{M}^{*}$ in $H^{s, p}$. The spaces $H^{s, p}, H_{ \pm}^{s, p}$, and $H^{s, p}\left(R_{ \pm}^{n}\right)$ were defined in $\$ 1$ for every real $s$ :

$$
\text { If } J^{s}=F^{-1}\left(1+|\xi|^{2}+\eta^{2}\right)^{s / 2} F, \text { then }\|u\|_{s, p}=\left\|J^{s} u\right\|_{L^{p}} .
$$

More generally $J^{t}$ maps $H^{s, p}$ onto $H^{s-t, p}$. The definitions make sense for complex $s$ too, however, since $\left(1+|\xi|^{2}+\eta^{2}\right)^{i \tau}$, $\tau$ real is an $L^{p}$-multiplier:

$$
\text { The Banach spaces } H^{s, p} \text { and } H^{s+i \tau, p} \text { are isomorphic, }
$$

and can be identified. We introduce the operators

$$
J_{ \pm}^{t}=F^{-1}\left(\eta \pm i\left(|\xi|^{2}+1\right)^{1 / 2}\right)^{t} F
$$

Both are invertible, and map $H^{s, p}$ onto $H^{s-t, p}$, so that the three norms $\left\|J^{s} u\right\|_{L^{p}}$, $\left\|J_{ \pm}^{s} u\right\|_{L^{p}}$ are equivalent, each can serve as a norm of $H^{s, p}$. By the Paley-Wiener theorem, $J_{-}^{t}$ maps $H_{-}^{s, p}$ onto $H_{-}^{s-t, p}$; i.e., $v_{-}=J_{-}^{t} u_{-}$is again supported in $R_{-}^{n}$ and

$$
\left\|u_{-}\right\|_{s, p} \sim\left\|J_{-}^{t} u_{-}\right\|_{s-t, p} \quad\left(=\left\|J_{-}^{s} u_{-}\right\|_{L^{p}} \text { if } s=t\right)
$$

( $\sim$ denotes equivalence of norms). It follows also that $J_{-}^{t}$ induces a map of the quotient space $H^{s, p}\left(R_{+}^{n}\right)$ onto $H^{s-t, p}\left(R_{+}^{n}\right)$ and

$$
\left\|Y_{+} u\right\|_{s, p} \sim\left\|Y_{+} J_{-}^{t} u\right\|_{s-t, p} \quad\left(=\left\|Y_{+} J_{-}^{s} u\right\|_{L^{p}} \text { if } s=t\right) .
$$

Recall that $Y_{+}$is the projection $H^{s, p} \rightarrow H^{s, p}\left(R_{+}^{n}\right)$ which for $s=0$ (but not in general) is given by multiplication by the characteristic function of $R_{+}^{n}$. Similar statements and formulas hold with + and - interchanged.

It is easily verified that an $L^{p}$ multiplier $m$ gives rise to a bounded operator $F^{-1} m F$ in all $H^{s, p}$. In particular this is true for our homogeneous matrices $M(\xi, \eta)$.

Let $s \geqq 0$. Let $s=k+\sigma$ where $k=[s]=$ the integral part of $s$ and $0 \leqq \sigma<1$. Let

$$
M_{s}(\xi, \eta)=(\eta-i|\xi|)^{s} M(\xi, \eta)(\eta+i|\xi|)^{-s}=M_{\sigma}(\xi, \eta)\left(\frac{\eta-i|\xi|}{\eta+i|\xi|}\right)^{k}
$$


(all factors commute since all are scalars except $M$ ). We have

$$
\begin{aligned}
(\eta-i|\xi|)^{\sigma}(\eta+i|\xi|)^{-\sigma} & \rightarrow e^{-2 \pi i \sigma} & & \text { as } \eta \rightarrow-\infty \\
& \rightarrow 1 & & \text { as } \eta \rightarrow+\infty
\end{aligned}
$$

while $(\eta-i|\xi|)^{k}(\eta+i|\xi|)^{-k} \rightarrow 1$ as $\eta \rightarrow \pm \infty$. Considering (4.1), (4.3), (4.5), and (4.12), we see the following: If $\zeta_{j}, \zeta_{j}^{\sigma}, \zeta_{j}^{s}$ are the fractional indices and $\kappa_{j}, \kappa_{j}^{(\sigma)}, \kappa_{j}^{(s)}$ the partial indices (for some $\xi$ ) in the factorization of $M(\xi, \eta), M_{\sigma}(\xi, \eta)$, and $M_{s}(\xi, \eta)$ respectively, then

$$
\begin{aligned}
\zeta_{j}^{(s)}=\zeta_{j}^{(\sigma)}=\zeta_{j} & +\sigma \quad(\bmod 1) . \\
\kappa_{j}^{(s)}=k+\kappa_{j}^{(\sigma)} & =k+\kappa_{j} \quad \text { if } \operatorname{Re} \zeta_{j}+\sigma \leqq 1 / p, \\
& =k+\kappa_{j}+1 \text { if } \operatorname{Re} \zeta_{j}+\sigma>1 / p .
\end{aligned}
$$

In particular if $\lambda_{j}^{(s)}$ are the eigenvalues relevant for $M_{s}$ then

$$
\operatorname{Arg} \lambda_{j}^{(s)} \neq 2 \pi / p \text { iff } \operatorname{Arg} \lambda_{j} \neq 2 \pi(1 / p+\sigma) .
$$

As usual, we set $M_{s}=F^{-1} M_{s} F$.

THEOREM 6.1. The following estimates are equivalent

$$
\begin{aligned}
& \|u\|_{s, p} \leqq C\left[\left\|Y_{-} u\right\|_{s, p}+\left\|Y_{+} M u\right\|_{s, p}\right], \quad u \in H^{s, p}, \\
& \|v\|_{o, p} \leqq C\left[\left\|Y_{-} v\right\|_{o, p}+\left\|Y_{+} J_{-}^{s} M J_{+}^{-s} v\right\|_{o, p}\right], \quad v \in L^{p}, \\
& \left\|Y_{+} v\right\|_{o, p} \leqq C\left\|Y_{+} M_{s} Y_{+} v\right\|, \quad v \in L^{p} .
\end{aligned}
$$

Proof. $u \in H^{s, p}$ if and only if $v=J_{+}^{s} u \in L^{p}$ and $\|u\|_{s, p}=\|v\|_{o, p}$. Using this substitution and (6.5) (for + and - ), we see that (6.11) is equivalent to (6.12). Now we are in $L^{p}$, and $v=Y_{+} v+Y_{-} v$. If $Y_{-} v=0$, then $v=Y_{+} v$ and (6.12) implies

$$
\left\|Y_{+} v\right\|_{o, p} \leqq C\left\|Y_{+} J_{-}^{s} M J_{+}^{-s} Y_{+} v\right\|_{o, p}, \quad v \in L^{p} .
$$

Now the operator $J_{-}^{s} M_{+}^{-s}$ corresponds in the Fourier transform to multiplication by the following symbol for $\lambda=1$ :

$$
\left(\eta-i\left(|\xi|^{2}+\lambda^{-2}\right)^{1 / 2}\right)^{s} M(\xi, \eta)\left(\eta+i\left(|\xi|^{2}+\lambda^{-2}\right)^{1 / 2}\right)^{-s} .
$$

Writing down (6.14) for $v(\lambda x, \lambda y)$ instead of $v(x, y)$ we get after a simple computation a similar estimate with (6.15) as symbol. But (6.15) tends to $M_{s}(\xi, \eta)$ as $\lambda \rightarrow \infty$ in the norm of $L^{p}$-multipliers, as one easily verifies (using Theorem 2.1). Thus (6.13) and (6.12) are equivalent and the proof is complete.

In particular for $M=1$ we proved in [12], [11], essentially by reduction to the 1-dimensional case, that for $s \geqq 0$

$$
\|u\|_{s, p} \leqq C\left[\left\|Y_{-} u\right\|_{s, p}+\left\|Y_{+} u\right\|_{s, p}\right] \text { iff } s \neq 1 / p \quad(\bmod 1) .
$$

This means that the map $u \rightarrow\left(Y_{-} u, Y_{+} u\right)$ of $H^{s, p} \rightarrow H^{s, p}\left(R_{-}^{n}\right) \times H^{s, p}\left(R_{+}^{n}\right)$ is $1-1$ and has closed range. It is known to have dense range for $0 \leqq s \leqq 1 / p$ [6]. Hence 
it is $1-1$ and onto for $0 \leqq s<1 / p$, and by conjugating we see that it is $1-1$ and onto for $-1+1 / p<s<1 / p$. In particular we get (6.16) true for $s \geqq-1+1 / p$. Observe now that the operator whose symbol is $1_{x+\zeta}$ is the (scalar) operator $D_{x+\zeta}$ of (5.2). We have now

COROllaRY 6.2. Let $\kappa \geqq 0$ be integer and $-1+1 / p \leqq \operatorname{Re} \zeta \leqq 1 / p$. Then

$$
\left\|Y_{+} u\right\|_{L^{p}} \leqq C\left\|Y_{+} D_{x+\zeta} Y_{+}\right\|_{L^{p}} \text { iff } \operatorname{Re} \zeta \neq 1 / p(\bmod 1) .
$$

Proof. For real $\zeta$ this follows from (6.16) (for $s \geqq-1+1 / p$ ) and Theorem 6.2 (the equivalence of (6.11) and (6.13)). For complex $\zeta$ one uses the observation (6.2).

Notice that Corollary 6.2 proves (5.4) and the first half of (5.5) follows from the first half (cf. Remark 5.2, end of §5).

Proof of Theorem A. The operator $\tilde{M}$ acting in $H^{k+\sigma, p}(s=k+\sigma)$ is 1-1 and has a closed range if and only if the estimate (6.17) is valid. By Theorem 6.1, this is the case if and only if (6.13) holds, i.e., if $Y_{+} M_{s} Y_{+}$is $1-1$ and has a closed range. But $\boldsymbol{M}_{s}=\left(\boldsymbol{M}_{\sigma}\right)_{k}=\boldsymbol{M}_{\sigma+k}$, we can apply Theorem B for $\boldsymbol{M}_{\sigma}$ and get the result, noticing that by (6.10) we indeed get the eigenvalues condition for $\sigma$.

We turn now to the second assertion of Theorem A about $\tilde{M}$ being onto for $s \rightarrow-\infty$. As in $\S 5$, we consider the adjoint of $\tilde{M}$ (for $s=k+\sigma$ ) acting between the adjoint spaces. It is given by

$$
\begin{array}{ll}
\tilde{M}^{*}: H_{-}^{-s, p^{\prime}} \times H_{+}^{-s, p^{\prime}} \rightarrow H^{-s, p^{\prime}}, & s=k+\sigma \\
\tilde{M}^{*}\left(V_{-}, V_{+}\right)=V_{-}+M^{*} V_{+}, & V_{ \pm} \in H_{ \pm}^{-s, p^{\prime}} .
\end{array}
$$

The question is then, when is $\tilde{M}^{*} 1-1$ and of closed range, or when does

$$
\sum_{ \pm}\left\|V_{ \pm}\right\|_{-s, p} \leqq C\left\|V_{-}+M^{*} V_{+}\right\|_{-s, p^{\prime}}, \quad V_{ \pm} \in H_{ \pm}^{-s, p^{\prime}}
$$

hold? By (6.4), $V_{ \pm} \in H_{ \pm}^{-s, p^{\prime}}$ iff $V_{ \pm}=J_{ \pm}^{s} v_{ \pm}, v_{ \pm} \in L_{ \pm}^{p^{\prime}}$ and $\left\|V_{ \pm}\right\|_{-s, p^{\prime}}=\left\|v_{ \pm}\right\|_{L^{p^{\prime}}}$. Hence we easily get that (6.19) is equivalent to the $L^{p \prime}$ estimate

$$
\left\|v_{-}\right\|+\left\|v_{+}\right\| \leqq C\left\|v_{-}+\left(M^{*}\right)_{-s} v_{+}\right\|, \quad v_{ \pm} \in L_{ \pm}^{p^{\prime}}
$$

which in turn is equivalent to

$$
\left\|Y_{+} v\right\|_{L^{p^{\prime}}} \leqq C\left\|Y_{+}\left(M^{*}\right)_{-8} Y_{+} v\right\|_{L^{p^{\prime}}}, \quad v \in L^{p^{\prime}}
$$

Here again we can apply Theorem B. Since $\left(M^{*}\right)_{-s}=\left(M^{*}\right)_{-\sigma-k}$ and

$$
\operatorname{Re}\left(-\zeta_{j}-\sigma\right) \neq 1 / p^{\prime}(\bmod 1) \quad \text { iff } \quad \operatorname{Re} \zeta_{j}+\sigma \neq 1 / p(\bmod 1),
$$

which is again (6.10), we see that the relevant eigenvalues condition is the one for $\sigma$. Finally $-k$ is sufficiently large iff $k^{\prime} \leqq k^{\prime \prime}$. This concludes the proof.

Proofs of Theorems $\mathbf{A}^{\prime}, \mathbf{B}^{\prime}$ and Corollary $\mathbf{A}^{\prime \prime}$. We first prove $\mathscr{N}^{s+l, p}=J^{-l} \mathscr{N}^{s, p}$ $\cap J_{+}^{-l} \mathscr{N}^{s, p}$. If $u \in \mathscr{N}^{s+l, p}$ then both $v_{1}=J_{-}^{l} u$ and $v_{2}=J_{+}^{l} u$ belong to $\mathscr{N}^{s, p}$, since $J_{ \pm}^{l}$ 
have holomorphic symbols. This proves left-to-right inclusion. Conversely assume that $u=J_{-}^{-l} v_{1}=J_{+}^{-l} v_{2}, v_{1}, v_{2} \in \mathscr{N}^{s, p}$. From $Y_{-} v_{2}=0$ we get $Y_{-} J_{+}^{-l} v_{2}=Y_{-} u=0$. From $Y_{+} M v_{1}=0$ we get $Y_{+} M J_{-}^{-l} v_{1}=Y_{+} M u=0$. Thus $u \in \mathscr{N}^{s+l, p}$.

Next we prove $\mathscr{R}_{*}^{s-l, p}=J_{-}^{l} \mathscr{R}_{*}^{s, p}+J^{l} \mathscr{R}_{*}^{s, p}$. The right-to-left inclusion is obvious. Now if $U_{-}+M^{*} U_{+}=W \in \mathscr{R}_{*}^{s-l, p}$ then $U_{-}=\tilde{M}^{*}\left(U_{-}, 0\right)$ and $M^{*} U_{+}=\tilde{M}^{*}\left(0, U_{+}\right)$ while both

$$
J_{-}^{-l} U_{-}=\tilde{M}^{*}\left(J_{-}^{-l} u_{-}, 0\right), \quad J_{+}^{-l} M^{*} U_{+}=\tilde{M}^{*}\left(0, J_{+}^{-l} U_{+}\right) \in R_{*}^{s+l, p},
$$

proving the result. Similar relations can be proved for $\mathscr{R}^{s, p}$ and $\mathscr{N}_{*}^{s, p}$ (directly, or by duality). However the (recursive) relation for the range of $\tilde{M}^{*}$ proves that it is closed for $s-l$ if and only if it is closed for $s$. The same is true for the range of $\tilde{M}$ and this obviously proves Corollary $\mathrm{A}^{\prime \prime}$. Theorem $\mathrm{B}^{\prime}$ is obtained too since the range of $M$ is closed in $L^{p}\left(R_{+}^{n}\right)$ iff the range of $\tilde{M}$ is closed for $s=0$.

7. Appendix. We shall prove here the factorization Theorem 4.4 for $M(\xi, \eta)$ in (4.3) is not necessarily satisfied, i.e., the "jump matrix" $E$ is nondiagonizable. The only change will be in the definition (but not the properties) of $Q_{ \pm}$, due to the more complicated "fill in" process.

As in $\$ 4, \lambda_{j}, 1 \leqq j \leqq N$, are the eigenvalues of $E$ and $\zeta_{j}$ are given by (4.5). We can assume that $E$ is in a suitable canonical form, achieved by similarity transformation. Jordan's form is not suitable for our purposes. However, it is easily verified that a Jordan block (with $\lambda$ along its main diagonal and 1's along a subdiagonal) is similar to a square matrix $\lambda B(1)$ of the same order where $B(\alpha)\left(B^{r}(\alpha)\right.$ if the order $r$ is specified) is given by

$$
\begin{aligned}
{[B(\alpha)]_{j k} } & =0, & j<k, & B(0)=I, \\
& =\alpha^{j-k} /(j-k) !, & j>k, &
\end{aligned}
$$

Thus we may assume that

$$
E_{+}=I, E_{-}=\operatorname{diag}\left[\mu_{1} B^{r_{1}}(1), \ldots, \mu_{m} B^{r_{m}}(1)\right]
$$

where $\mu_{1}, \ldots, \mu_{m}$ exhaust all the eigenvalues $\lambda_{1}, \ldots, \lambda_{N}$.

A simple computation shows that $B(\alpha+\beta)=B(\alpha) B(\beta)$. In particular $B(-\alpha)$ $=[B(\alpha)]^{-1}$. We define now $\alpha_{ \pm}(\eta)=(2 \pi i)^{-1} \log (\eta \pm i)$. Then

$$
\begin{aligned}
\alpha_{-}(\eta)-\alpha_{+}(\eta) & \rightarrow 0, \eta \rightarrow \infty, \quad \text { so } B\left(\alpha_{-}(\eta)\right) B^{-1}\left(\alpha_{+}(\eta)\right) \rightarrow I \\
& \rightarrow-1, \eta \rightarrow-\infty, \text { so } B\left(\alpha_{-}(\eta)\right) B^{-1}\left(\alpha_{+}(\eta)\right) \rightarrow[B(1)]^{-1}
\end{aligned}
$$

Corresponding to the block decomposition of $E_{-}$in (7.2) we set

$$
B_{ \pm}(\eta)=\operatorname{diag}\left[B^{r_{1}}\left(a_{ \pm}\right), \ldots, B^{r_{m}}\left(\alpha_{ \pm}\right)\right] .
$$

Then $B_{+}(\eta)\left[B_{-}(\eta)\right]$ is holomorphic in $\operatorname{Im} \eta>0$ [Im $\left.\eta<0\right]$. Moreover they commute with the diagonal matrix $(\eta \pm i)^{\zeta}$ since the eigenvalue (hence the component of $\zeta$ ) 
has the same value inside a block of $E$, so $(\eta \pm i)^{\zeta}$ acts as a scalar on each block of $B_{ \pm}(\eta)$. We set now (cf. (4.7))

$$
M_{*}(\xi, \eta)=(\eta-i)^{-\zeta} B_{-}(\eta) M(\xi, \eta) B_{+}^{-1}(\eta)(\eta+i)^{\zeta} .
$$

In view of (7.3) and (7.4), $B_{-} M B_{+}^{-1}$ will tend to $I$ at $+\infty$ and to diag $\left[\lambda_{1}, \ldots, \lambda_{N}\right]$ at $\eta=-\infty$. Therefore, as in $\S 4, M_{*}$ will tend to $I$ as $\eta \rightarrow \pm \infty$, so the jump of $M$ is filled. In fact we have, corresponding to Lemma 4.3.

LEMMA 7.1. If $M \in C^{l+1}\left(S^{n-1}\right)$ is invertible then (4.8), (4.9), and (4.11) hold with any $\delta^{\prime}<\delta$.

Proof. The present $M_{*}$ is very much like $M_{*}$ of $\$ 4$ except that it contains the factors $B_{ \pm}(\eta)$ with logarithmic entries. Their presence does not change the proof of the estimates (4.8), (4.9), (4.11) except that $\delta$ has to be replaced by $\delta^{\prime}<\delta$.

Again by the last assertion of Lemma 4.3, $M_{*} \in O R_{N}^{l}$ and can be factored as in Theorem 3.1. For $M$ itself we get

TheOREM 7.2. Let $M(\xi, \eta) \in C^{l+1}\left(S^{n-1}\right)$ be homogeneous and invertible for any fixed $\xi$ we have the factorization (4.12) with

$$
Q_{ \pm}(\eta)=B_{ \pm}(\eta)(\eta \pm i)^{\zeta} N_{ \pm}(\eta)(\eta \pm i)^{-\zeta}
$$

The estimates (4.14) and (4.17) hold with $\delta^{\prime}<\delta$ (due to the logarithmic factors) and all the remaining assertions of Theorem 4.4 and Corollary 4.6 hold verbatim.

\section{BIBLIOGRAPHY}

1. A. P. Calderon, Intermediate spaces and interpolation, the complex method, Studia Math. 24 (1964), 113-190.

2. I. C. Gohberg, Factorization problems in normed rings, Uspehi Mat. Nauk 19 (1964), 71-124.

3. I. C. Gohberg and M. G. Krein, Systems of singular integral equations on the half line with kernels depending on the arguments, Uspehi Mat. Nauk 13 (1958), 3-72.

4. L. Hörmander, Estimates for translation invariant operators, Acta Math. 104 (1960), 93-140.

5. J. J. Kohn and L. Nirenberg, An algebra of pseudo-differential operators, Comm. Pure Appl. Math. 18 (1965), 269-305.

6. J. L. Lions and E. Magenes, Problèmes aux limites non homogènes (IV), Ann. Scuola Norm. Sup. Pisa 15 (1961), 311-326.

7. J. L. Lions and J. Peetre, Sur une class d'espaces d'interpolation, Inst. Hautes Études Sci. 19 (1964), 5-68.

8. J. Peetre, Mixed problems for higher order elliptic equations in two dimensions. I, Ann. Scuola Norm. Sup. Pisa 15 (1961), 337-353; II, ibid. 17 (1963), 1-12.

9. M. Schechter, Mixed problems for higher order elliptic partial differential equations, Comm. Pure Appl. Math. 13 (1960), 407-425.

10. E. Shamir, Mixed boundary value problems for elliptic equations in the plane-The $L^{p}$ theory, Ann. Scuola Norm Sup. Pisa 17 (1963), 117-138.

11. - Reduced Hilbert transform and singular integral equations, J. Analyse Math. 12 (1964), 277-305. 
12. - Une proprieté des espaces $H^{s, p}$, C. R. Acad. Sci. Paris 255 (1962), 448-449.

13. - Multipliers of Fourier transforms in a half-space, Bull. Amer. Math. Soc. 71 (1965), 165-167.

14. - Wiener-Hopf type problems for elliptic systems of singular integral equations, Bull. Amer. Math. Soc. 72 (1966), 501-504.

15. —, A remark on Mikhlin Hörmander multipliers theorem, J. Math. Anal. Appl. (1966), (to appear).

16.,$- L^{p}$ solution of Riemann-Hilbert systems with piecewise continuous coefficients, Dokl. Akad. Nauk SSSR 167 (1966), 1000-1003 = Soviet Math. Dokl 7 (1966), 530-533.

17. E. C. Titchmarch, Introduction to the theory of Fourier integrals, 2nd ed., Oxford, at the Clarendon Press, 1948.

18. M. I. Visik and G. I. Èskin, General boundary value problems with discontinuous conditions at the boundary, Dokl. Akad. Nauk SSSR 158 (1964), 15-19=Soviet Math. Dokl. 5 (1964), 1154-1157.

19. - Convolution equations in bounded domains, Uspehi Mat. Nauk 20 (1965), 15-19.

20. - Convolution equations in bounded domains in spaces with weighted norms, Mat. Sb. 69 (1966), 65-110.

NORTHWESTERN UNIVERSTTY, Evanston, Illinois 